PROCEEDINGS OF THE

AMERICAN MATHEMATICAL SOCIETY

Volume 138, Number 6, June 2010, Pages 2015-2023

S 0002-9939(10)10344-X

Article electronically published on February 5, 2010

\title{
NOTES ON THE REGULARITY OF HARMONIC MAP SYSTEMS
}

\author{
TAO HUANG AND CHANGYOU WANG
}

(Communicated by Chuu-Lian Terng)

\begin{abstract}
In this paper, we provide an alternative proof of $C^{1, \alpha}$-regularity of continuous weak solutions to the system of harmonic maps or heat flow of harmonic maps by Riesz potential estimates between Morrey spaces.
\end{abstract}

\section{INTRODUCTION}

It is well-known that the higher order regularity theory asserts any continuous weak solution to both the system of harmonic maps and heat flow of harmonic maps is smooth (see [9], 10]). The classical technique in the literature $([9],[10]$ ) is the hole filling argument, which enables us to establish (i) $C^{0}$-regularity implies $C^{\alpha}$-regularity for all $\alpha \in(0,1)$, and (ii) $C^{\alpha}$-regularity implies $C^{1, \beta}$-regularity for some $\beta \in(0,1)$. See Schoen [12] for another proof. In this paper, we will give an alternative proof of (ii) by the application of Riesz potential estimates between Morrey spaces. Such an estimate was due to Adams [1] in $\mathbb{R}^{n}$ with the Euclidean metric. Here we extend such an estimate to $\mathbb{R}^{n}$ with the parabolic metric, in order to handle the equation of harmonic map heat flows.

Now we recall the notions of Riesz potential and Morrey space on $\mathbb{R}^{n}$. Let $d(x, y): \mathbb{R}^{n} \times \mathbb{R}^{n} \rightarrow \mathbb{R}_{+}$be a distance function on $\mathbb{R}^{n}$. Let $\hat{n}$ denote the Hausdorff dimension of $\mathbb{R}^{n}$ with respect to the distance $d$. On $\left(\mathbb{R}^{n}, d\right)$, we define

Definition 1.1. For any $f \in L^{p}\left(\mathbb{R}^{n}\right), 1 \leq p<+\infty$, and $0<\beta \leq \hat{n}$, the Riesz potential of $f$ of order $\beta$ is defined by

$$
I_{\beta}(f)(x) \equiv \int_{\mathbb{R}^{n}} \frac{|f(y)|}{d(x, y)^{\hat{n}-\beta}} d y, \quad x \in \mathbb{R}^{n} .
$$

For $x \in \mathbb{R}^{n}$ and $r>0$, let $B_{r}^{d}(x)=\left\{y \in \mathbb{R}^{n}: d(y, x) \leq r\right\}$ be the ball with center $x$ and radius $r$ with respect to the distance $d$.

Definition 1.2. For $\Omega \subset \mathbb{R}^{n}, 1 \leq p<+\infty$, and $0 \leq \lambda \leq \hat{n}$, the Morrey space $M^{p, \lambda}(\Omega)$ is defined to be the collection of functions $f \in L^{p}(\Omega)$ satisfying

$$
\|f\|_{M^{p, \lambda}(\Omega)} \equiv \sup _{r>0, x \in \Omega}\left(r^{\lambda-\hat{n}} \int_{\Omega \cap B_{r}^{d}(x)}|f(y)|^{p} d y\right)^{\frac{1}{p}}<\infty .
$$

Received by the editors June 12, 2009.

2010 Mathematics Subject Classification. Primary 35J50, 35K40; Secondary 58E20.

This work was partially supported by NSF grant 0601162 .

(C)2010 American Mathematical Society 
For the purpose of this paper, we also need to recall the weak Morrey space.

Definition 1.3. For $\Omega \subset \mathbb{R}^{n}$ and $1 \leq p<+\infty$, the weak $L^{p}$ space $L^{p, *}(\Omega)$ is defined to be the collection of measurable functions $f$ on $\Omega$ such that

$$
\|f\|_{L^{p, *}(\Omega)}^{p} \equiv \sup _{t>0} t^{p}|\{x \in \Omega|| f(x) \mid>t\}|<+\infty .
$$

For $0 \leq \lambda \leq \hat{n}$, the weak Morrey space $M_{*}^{p, \lambda}(\Omega)$ is defined to be the set of functions $f \in L^{p, *}(\Omega)$ satisfying

$$
\|f\|_{M_{*}^{p, \lambda}(\Omega)}^{p} \equiv \sup _{x \in \Omega, r>0}\left\{r^{\lambda-\hat{n}}\|f\|_{L^{p, *}\left(\Omega \cap B_{r}^{d}(x)\right)}^{p}\right\}<+\infty .
$$

For the Euclidean distance $d(x, y)=|x-y|$ on $\mathbb{R}^{n}$, we have $\hat{n}=n$ and simply denote $B_{r}(x)$ as $B_{r}^{d}(x)$.

In this paper, we consider the higher order regularity issues to the following two systems:

(i) A map $u \in H^{1}\left(\Omega, \mathbb{R}^{m}\right)$ (for some $m \geq 1$ ) solves

$$
-\triangle u=Q(u)(\nabla u, \nabla u) \text { in } \Omega,
$$

where $Q \in C^{\infty}\left(\mathbb{R}^{m} \times\left(\mathbb{R}^{n m}\right)^{2}, \mathbb{R}^{m}\right)$ has quadratic growth in the last two variables:

$$
|Q(p)(v, w)| \leq C(R)|v||w|, \forall p \in \mathbb{R}^{m} \text { with }|p| \leq R, \text { and } v, w \in \mathbb{R}^{n m}
$$

for some positive constant $C=C(R)$ for any $R>0$. It is readily seen that (1.5) includes the equation of harmonic maps from $\Omega$ to a Riemannian manifold $(N, h) \subset \mathbb{R}^{m}$.

(ii) A map $u \in H^{1}\left(\Omega \times[0, T], \mathbb{R}^{m}\right)(T>0)$ solves

$$
u_{t}-\triangle u=Q(u)(\nabla u, \nabla u) \text { in } \Omega \times(0, T),
$$

where $Q$ enjoys property (1.6). It is also readily seen that (1.7) includes the heat flow of harmonic maps from $\Omega$ to a Riemannian manifold $(N, h) \subset \mathbb{R}^{m}$.

In this paper, we provide an alternative approach, based on the Riesz potential estimate between Morrey spaces, to the following two theorems. Both theorems are classical results in the literature (see [9], 10, 11]).

Theorem 1.4. There exists $\epsilon_{0}>0$ such that if $u(x) \in H^{1}\left(\Omega, \mathbb{R}^{m}\right)$ is a weak solution of (1.5)-(1.6) and satisfies, for $x_{0} \in \Omega$ and $0<r_{0}<\operatorname{dist}\left(x_{0}, \partial \Omega\right)$,

$$
\operatorname{Osc}_{B_{r_{0}}\left(x_{0}\right)} u \leq \epsilon_{0},
$$

then $u \in C^{\infty}\left(B_{\frac{r_{0}}{2}}\left(x_{0}\right), \mathbb{R}^{m}\right)$.

Theorem 1.5. There exists $\epsilon_{0}>0$ such that if $u(x) \in H^{1}\left(\Omega \times[0, T], \mathbb{R}^{m}\right)$ is $a$ weak solution of (1.7) and (1.6) and satisfies, for $\left(x_{0}, t_{0}\right) \in \Omega \times(0, T)$ and $0<r_{0}<\min \left\{\operatorname{dist}\left(x_{0}, \partial \Omega\right), \sqrt{t_{0}}\right\}$,

$$
\operatorname{osc}_{B_{r_{0}}\left(x_{0}\right) \times\left[t_{0}-r_{0}^{2}, t_{0}+r_{0}^{2}\right]} u \leq \epsilon_{0},
$$

then $u \in C^{\infty}\left(B_{\frac{r_{0}}{2}}\left(x_{0}\right) \times\left[t_{0}-\frac{r_{0}^{2}}{4}, t_{0}+\frac{r_{0}^{2}}{4}\right], \mathbb{R}^{m}\right)$.

For both minimizing harmonic maps and stationary harmonic maps (or stationary heat flow of harmonic maps), the small oscillation condition (1.8) (or (1.9) ) can be deduced by the small renormalized energy condition (see [8], 13], 4], 2], 7], 3], [6]). In general, for weak harmonic maps (or weak heat flow of harmonic maps), (1.8) (or (1.9) ) can only be guaranteed if $K_{N} \leq 0$ or $u\left(B_{r_{0}}\left(x_{0}\right)\right)$ is contained in a geodesic convex ball (see [5], [10, [11]). 


\section{Proof of Theorem 1.4}

In this section, we will apply the Riesz potential estimate between Morrey spaces to prove Theorem 1.4. This can also serve as a guideline to prove Theorem 1.5. The proof of the following lemma can be found in Adams [1].

Lemma 2.1. Let $d(x, y)=|x-y|$ be the Euclidean distance on $\mathbb{R}^{n}$.

(i) For any $\beta>0,0<\lambda \leq n, 1<p<\frac{\lambda}{\beta}$, if $f \in L^{p}\left(\mathbb{R}^{n}\right) \cap M^{p, \lambda}\left(\mathbb{R}^{n}\right)$, then $I_{\beta}(f) \in L^{\tilde{p}}\left(\mathbb{R}^{n}\right) \cap M^{\tilde{p}, \lambda}\left(\mathbb{R}^{n}\right)$, where $\tilde{p}=\frac{\lambda p}{\lambda-p \beta}$. Moreover,

$$
\begin{aligned}
\left\|I_{\beta}(f)\right\|_{L^{\tilde{p}}\left(\mathbb{R}^{n}\right)} & \leq C\|f\|_{M^{p, \lambda}\left(\mathbb{R}^{n}\right)}^{\frac{\beta p}{\lambda}}\|f\|_{L^{p}\left(\mathbb{R}^{n}\right)}^{1-\frac{\beta p}{(}}, \\
\left\|I_{\beta}(f)\right\|_{M_{\tilde{p}, \lambda}\left(\mathbb{R}^{n}\right)} & \leq C\|f\|_{M^{p, \lambda}\left(\mathbb{R}^{n}\right)} .
\end{aligned}
$$

(ii) For $0<\beta<\lambda \leq n$, if $f \in L^{1}\left(\mathbb{R}^{n}\right) \cap M^{1, \lambda}\left(\mathbb{R}^{n}\right)$, then $I_{\beta}(f) \in L^{\frac{\lambda}{\lambda-\alpha}, *}\left(\mathbb{R}^{n}\right) \cap$ $M_{*}^{\frac{\lambda}{\lambda-\alpha}, \lambda}\left(\mathbb{R}^{n}\right)$. Moreover,

$$
\begin{aligned}
\left\|I_{\beta} f\right\|_{L^{\frac{\lambda}{\lambda-\beta}, *}\left(\mathbb{R}^{n}\right)} & \leq C\|f\|_{M^{1, \lambda}\left(\mathbb{R}^{n}\right)}^{\frac{\beta}{\beta}}\|f\|_{L^{1}\left(\mathbb{R}^{n}\right)}^{1-\frac{\beta}{1}}, \\
\left\|I_{\beta}(f)\right\|_{M_{*}^{\frac{\lambda}{\lambda-\beta}, \lambda}\left(\mathbb{R}^{n}\right)} & \leq C\|f\|_{M^{1, \lambda}\left(\mathbb{R}^{n}\right)} .
\end{aligned}
$$

Proof of Theorem 1.4.

Claim 1. $\nabla u \in M^{2,2-2 \alpha}\left(B_{\frac{r_{0}}{2}}\left(x_{0}\right)\right)$ for any $\alpha \in(0,1)$.

To see it, let $B_{r}(x) \subset B_{r_{0}}\left(x_{0}\right)$ be any ball. Let $v: B_{r}(x) \rightarrow \mathbb{R}^{m}$ solve

$$
\Delta v=0 \text { in } B_{r}(x), v=u \text { on } \partial B_{r}(x) .
$$

Multiplying both (1.5) and (2.5) by $u-v$, integrating over $B_{r}(x)$, we get

$$
\int_{B_{r}(x)}|\nabla(u-v)|^{2} \leq C \int_{B_{r}(x)}|\nabla u|^{2}|u-v| \leq C \epsilon_{0} \int_{B_{r}(x)}|\nabla u|^{2},
$$

where the last inequality follows from the maximum principle of $v$ :

$$
\sup _{B_{r}(x)}|u-v| \leq \operatorname{osc}_{B_{r}(x)} u \leq \epsilon_{0}
$$

Hence, for any $\theta \in(0,1)$,

$$
\begin{aligned}
(\theta r)^{2-n} \int_{B_{\theta r}(x)}|\nabla u|^{2} & \leq(\theta r)^{2-n} \int_{B_{\theta r}(x)}|\nabla v|^{2}+\theta^{2-n} r^{2-n} \int_{B_{r}(x)}|\nabla(u-v)|^{2} \\
& \leq C\left(\theta^{2}+\epsilon_{0} \theta^{2-n}\right) r^{2-n} \int_{B_{r}(x)}|\nabla u|^{2}
\end{aligned}
$$

where we have used the following inequality for $v$ :

$$
(\theta r)^{2-n} \int_{B_{\theta r}(x)}|\nabla v|^{2} \leq C \theta^{2} r^{2-n} \int_{B_{r}(x)}|\nabla v|^{2} .
$$

For any $\alpha \in(0,1)$, first choose $\theta=\theta_{0}(\alpha) \in(0,1)$ such that $C \theta_{0}^{2} \leq \frac{1}{2} \theta_{0}^{2 \alpha}$ and then choose $\epsilon_{0}$ such that $C \theta_{0}^{2-n} \epsilon_{0} \leq \frac{1}{2} \theta_{0}^{2 \alpha}$. We obtain

$$
\left(\theta_{0} r\right)^{2-n} \int_{B_{\theta_{0} r}(x)}|\nabla u|^{2} \leq \theta_{0}^{2 \alpha} r^{2-n} \int_{B_{r}(x)}|\nabla u|^{2} .
$$


Iterating this inequality finitely many times yields

$$
r^{2-n} \int_{B_{r}(x)}|\nabla u|^{2} \leq C r^{2 \alpha} r_{0}^{2-n} \int_{B_{r_{0}}\left(x_{0}\right)}|\nabla u|^{2}, \forall B_{r}(x) \subset B_{\frac{r_{0}}{2}}\left(x_{0}\right) .
$$

This implies Claim 1.

Claim 2. $\nabla u \in L^{q}\left(B_{\frac{r_{0}}{2}}\left(x_{0}\right)\right)$ for any $1<q<+\infty$.

For simplicity, assume $x_{0}=0$ and $r_{0}=4$. Let $\eta \in C_{0}^{\infty}\left(B_{4}(0)\right)$ be such that $0 \leq \eta(x) \leq 1, \eta(x) \equiv 1$ on $B_{2}(0)$, and $|\nabla \eta|+\left|\nabla^{2} \eta\right| \leq 8$. Set $v=\eta u$. Then $v$ satisfies

$$
-\triangle v(x)=H(x), H(x)=Q(u)(\nabla u, \nabla u) \eta-\left(u-u_{2}\right) \triangle \eta-2 \nabla u \nabla \eta,
$$

where $u_{2}=\frac{1}{\left|B_{2}(0)\right|} \int_{B_{2}(0)} u$ is the average of $u$ over $B_{2}$. Let $G(x)$ be the fundamental solution of $\Delta$ on $\mathbb{R}^{n}$. Then we have

$$
v(x)=\int_{\mathbb{R}^{n}} G(x-y) H(y) d y
$$

so that

$$
\begin{aligned}
|\nabla v(x)| & =\left|\int_{\mathbb{R}^{n}} \nabla G(x-y) H(y) d y\right| \\
& \leq C(n) \int_{\mathbb{R}^{n}} \frac{|H(y)|}{|x-y|^{n-1}} d y \\
& =C(n) I_{1}(|H|)(x)
\end{aligned}
$$

for some positive constant $C(n)$.

Since $H=0$ outside $B_{4}(0)$, it is not difficult to see that $H \in M^{1,2-2 \alpha}\left(\mathbb{R}^{n}\right)$ and

$$
\begin{aligned}
\|H\|_{M^{1,2-2 \alpha}\left(\mathbb{R}^{n}\right)} & \leq C\|H\|_{M^{1,2-2 \alpha}\left(B_{4}(0)\right)} \\
& \leq C \sup _{B_{r}(x) \subset B_{4}(0)}\left\{r^{2-2 \alpha-n} \int_{B_{r}(x)}|H(y)| d y\right\} \\
& \leq C\left[\operatorname{osc}_{B_{4}(0)} u+\|\nabla u\|_{M^{2,2-2 \alpha}\left(B_{4}(0)\right)}\right] \leq C .
\end{aligned}
$$

Hence Lemma 2.1 implies that for any $0<\alpha<\frac{1}{2},|\nabla v| \in L^{\frac{2-\alpha}{1-2 \alpha}, *}\left(\mathbb{R}^{n}\right)$. Thus $\nabla u \in L^{\frac{2-\alpha}{1-2 \alpha}, *}\left(B_{2}(0)\right)$. Since $L^{\frac{2-\alpha}{1-2 \alpha}, *}\left(B_{2}(0)\right) \subset L^{q}\left(B_{2}(0)\right)$ for any $q<\frac{2-2 \alpha}{1-2 \alpha}$, we have $\nabla u \in L^{q}\left(B_{2}(0)\right.$ for any $q<\frac{2-2 \alpha}{1-2 \alpha}$. Since

$$
\lim _{\alpha \uparrow \frac{1}{2}} \frac{2-2 \alpha}{1-2 \alpha}=+\infty
$$

$\nabla u \in L^{q}\left(B_{2}(0)\right)$ for any $1 \leq q<\infty$. Applying the $W^{2, q}$-estimate of the equation (1.5), we conclude that $u \in W^{2, q}\left(B_{2}(0)\right)$ for any $q \in(1,+\infty)$. In particular, the Sobolev embedding theorem implies $\nabla u \in C^{\delta}\left(B_{2}(0)\right)$ for any $\delta \in(0,1)$. Now, we can apply the Schauder theory to show that $u \in C^{\infty}\left(B_{2}(0)\right)$.

\section{Proof of Theorem 1.5}

In this section, we will extend Adams' Riesz potential estimate to $\mathbb{R}^{n+1}$, equipped with the parabolic distance $\delta$, and then apply it to prove Theorem 1.5

First, let's recall the notion of parabolic distance $\delta$ on $\mathbb{R}^{n+1}$ :

$$
\delta((x, t),(y, s))=\max \{|x-y|, \sqrt{|t-s|}\}, \forall(x, t),(y, s) \in \mathbb{R}^{n+1} .
$$


Denote $P_{r}(x, t)=B_{r}^{\delta}(x, t)$, the ball centered at $(x, t)$ with radius $r$ with respect to the distance $\delta$. Notice that $P_{r}(x, t)=B_{r}(x) \times\left[t-r^{2}, t+r^{2}\right]$. For short, write $P_{r}=P_{r}(0,0)$.

Notice that the Hausdorff dimension of $\mathbb{R}^{n+1}$ with respect to the parabolic distance $\delta$ is $n+2$. Hence the Riesz potential of order $\beta \in[0, n+2]$ on $\mathbb{R}^{n+1}$ is given by

$$
\widetilde{I}_{\beta}(f)=\iint_{\mathbb{R}^{n+1}} \frac{|f(y, s)|}{\delta((x, t),(y, s))^{n+2-\beta}} d y d s, f \in L^{p}\left(\mathbb{R}^{n+1}\right),
$$

and the Morrey space $\widetilde{M}^{p, \lambda}\left(\Omega_{T}\right)$ (or the weak Morrey space $\widetilde{M}_{*}^{p, \lambda}\left(\Omega_{T}\right)$ respectively) on $\Omega_{T}=\Omega \times(0, T) \subset \mathbb{R}^{n+1}$, for $1 \leq p<+\infty$ and $0 \leq \lambda \leq n+2$, is the collection of functions $f$ on $\Omega_{T}$ such that

$$
\begin{gathered}
\|f\|_{\widetilde{M}^{p, \lambda}\left(\Omega_{T}\right)}^{p} \equiv \sup _{r>0, z \in \Omega_{T}} r^{\lambda-(n+2)}\|f\|_{L^{p}\left(\Omega_{T} \cap P_{r}(z)\right.}^{p}<\infty \\
\left(\text { or }\|f\|_{\widetilde{M}_{*}^{p, \lambda}\left(\Omega_{T}\right)}^{p} \equiv \sup _{r>0, z \in \Omega_{T}} r^{\lambda-(n+2)}\|f\|_{L^{p, *}\left(\Omega_{T} \cap P_{r}(z)\right.}^{p}<\infty\right. \text { respectively). }
\end{gathered}
$$

Now we are ready to prove the Riesz potential estimate on $\left(\mathbb{R}^{n+1}, \delta\right)$, namely,

Theorem 3.1. (i) For any $\beta>0,0<\lambda \leq n+2,1<p<\frac{\lambda}{\beta}$, if $f \in L^{p}\left(\mathbb{R}^{n+1}\right) \cap$ $\widetilde{M}^{p, \lambda}\left(\mathbb{R}^{n+1}\right)$, then $\widetilde{I}_{\beta}(f) \in L^{\tilde{p}}\left(\mathbb{R}^{n+1}\right) \cap \widetilde{M}^{\tilde{p}, \lambda}\left(\mathbb{R}^{n+1}\right)$, where $\tilde{p}=\frac{p \lambda}{\lambda-p \beta}$. Moreover,

$$
\begin{aligned}
\left\|\widetilde{I}_{\beta}(f)\right\|_{L^{\tilde{p}}\left(\mathbb{R}^{n+1}\right)} & \leq C\|f\|_{\widetilde{M}^{p, \lambda}\left(\mathbb{R}^{n+1}\right)}^{\frac{\beta p}{\lambda}}\|f\|_{L^{p}\left(\mathbb{R}^{n+1}\right)}^{1-\frac{\beta p}{(}}, \\
\left\|\widetilde{I}_{\beta}(f)\right\|_{\widetilde{M}^{\tilde{p}, \lambda}\left(\mathbb{R}^{n+1}\right)} & \leq C\|f\|_{\widetilde{M}^{p, \lambda}\left(\mathbb{R}^{n+1}\right)} .
\end{aligned}
$$

(ii) For $0<\beta<\lambda \leq n+2$, if $f \in L^{1}\left(\mathbb{R}^{n+1}\right) \cap \widetilde{M}^{1, \lambda}\left(\mathbb{R}^{n+1}\right)$, then $\widetilde{I}_{\beta}(f) \in$ $L^{\frac{\lambda}{\lambda-\beta}, *}\left(\mathbb{R}^{n+1}\right) \cap \widetilde{M}_{*}^{\frac{\lambda}{\lambda-\beta}, \lambda}\left(\mathbb{R}^{n+1}\right)$. Moreover,

$$
\begin{aligned}
& \left\|\widetilde{I}_{\beta}(f)\right\|_{L^{\frac{\lambda}{\lambda-\beta}, *}\left(\mathbb{R}^{n+1}\right)} \leq C\|f\|_{\widetilde{M}^{1, \lambda}\left(\mathbb{R}^{n+1}\right)}^{\frac{\beta}{\lambda}}\|f\|_{L^{1}\left(\mathbb{R}^{n+1}\right)}^{1-\frac{\beta}{2}},
\end{aligned}
$$

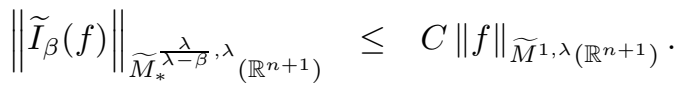

Proof. Assume $f \not \equiv 0$. Then

$$
\widetilde{I}_{\beta}(f)(x, t)=\iint_{P_{\epsilon}(x, t)} f+\iint_{\mathbb{R}^{n+1} \backslash P_{\epsilon}(x, t)} f:=I_{1}+I_{2},
$$

where $\epsilon>0$ is to be determined later. For $k \in \mathbb{Z}$, let

$$
A_{k}=\left\{(y, s) \mid(y, s) \in P_{2^{k+1} \epsilon}(x, t) \backslash P_{2^{k} \epsilon}(x, t)\right\} .
$$

Since $\beta \leq n+2$ and $\beta<\frac{\lambda}{p}$, we have

$$
\begin{aligned}
\left|I_{2}\right| & \leq \sum_{k=1}^{\infty} \iint_{A_{k}} \delta((x, t),(y, s))^{\beta-(n+2)}|f(y, s)| d y d s \\
& \leq \sum_{k=1}^{\infty}\left(2^{k} \epsilon\right)^{\beta-(n+2)}\left(2^{k+1} \epsilon\right)^{n+2-\frac{\lambda}{p}} \mathcal{M}_{\frac{\lambda}{p}} f(x, t) \\
& \leq C \epsilon^{\beta-\frac{\lambda}{p}} \mathcal{M}_{\frac{\lambda}{p}} f(x, t),
\end{aligned}
$$


where

$$
\mathcal{M}_{\gamma} g(x, t)=\sup _{r>0} r^{\gamma-(n+2)} \iint_{P_{r}(x, t)}|g(y, s)| d y d s
$$

for $0 \leq \gamma \leq n+2$. Notice that $\mathcal{M}_{0} g$ is the parabolic Hardy-Littlewood maximal function of $g$. Similarly, we have

$$
\begin{aligned}
\left|I_{1}\right| & \leq \sum_{k=1}^{\infty} \iint_{A_{-k}} \delta((x, t),(y, s))^{\beta-(n+2)}|f(y, s)| d y d s \\
& \leq \sum_{k=1}^{\infty}\left(2^{-k} \epsilon\right)^{\beta-(n+2)}\left(2^{-k+1} \epsilon\right)^{n+2} \mathcal{M}_{0}(f)(x, t) \\
& \leq C \epsilon^{\beta} \mathcal{M}_{0}(f)(x, t) .
\end{aligned}
$$

Since

$$
\mathcal{M}_{\frac{\lambda}{p}}(f)(x, t) \leq C\left[\mathcal{M}_{\lambda}\left(|f|^{p}\right)(x, t)\right]^{\frac{1}{p}} \leq C\|f\|_{\widetilde{M}^{p, \lambda}\left(\mathbb{R}^{n+1}\right)},
$$

we conclude, by choosing $\epsilon=\epsilon(x, t)=\left(\frac{\|f\|_{\widetilde{M}^{p}, \lambda\left(\mathbb{R}^{n+1}\right)}}{\mathcal{M}_{0}(f)(x, t)}\right)^{\frac{p}{\lambda}}$, that

$$
\left|\widetilde{I}_{\beta} f(x, t)\right| \leq C\left[\|f\|_{\widetilde{M}^{p, \lambda}\left(\mathbb{R}^{n+1}\right)}\right]^{\frac{\beta p}{\lambda}}\left[\mathcal{M}_{0} f(x, t)\right]^{1-\frac{\beta p}{\lambda}} .
$$

When $p>1$, both estimates (3.1) and (3.2) follow by the well-known $L^{p}$-estimate for $\mathcal{M}_{0}(f)$ (see [14]).

When $p=1$, for any $l>0$, we have

$$
\begin{aligned}
& \left|\left\{(x, t) \in \mathbb{R}^{n+1}:\left|\widetilde{I}_{\beta} f(x, t)\right|>C l\right\}\right| \\
& \leq\left|\left\{(x, t) \in \mathbb{R}^{n+1}: \mathcal{M}_{0}(f)(x, t)>\frac{l^{\frac{\lambda}{\lambda-\beta}}}{\|f\|_{\widetilde{M}^{1, \lambda}\left(\mathbb{R}^{n+1}\right)}^{\frac{\beta}{\lambda-\beta}}}\right\}\right| \\
& \leq C \frac{\|f\|_{\widetilde{M}^{1, \lambda}\left(\mathbb{R}^{n+1}\right)}^{\frac{\beta}{\lambda^{2}}}\|f\|_{L^{1}\left(\mathbb{R}^{n+1}\right)},}{l^{\frac{\lambda}{\lambda-\beta}}}
\end{aligned}
$$

where we have used the weak $L^{1}$-estimate of $\mathcal{M}_{0}$ ([14, page 5 , Theorem 1 ). This implies both (3.3) and (3.4).

In order to prove Theorem 1.5, we need the following simple estimate on the heat kernel $G$.

Lemma 3.2. Let

$$
\begin{aligned}
G(x, t) & =\frac{1}{(4 \pi t)^{\frac{n}{2}}} \exp \left(-\frac{|x|^{2}}{4 t}\right) & & \text { for } t>0, \\
& =0 & & \text { for } t \leq 0
\end{aligned}
$$

be the fundamental solution of the heat equation on $\mathbb{R}^{n}$. Then

$$
|\nabla G(x, t)| \leq \frac{C}{\delta((x, t),(0,0))^{n+1}}, \forall(x, t) \in \mathbb{R}^{n+1} .
$$

Proof. It suffices to show (3.5) for $t>0$. Direct calculations give

$$
\nabla G(x, t)=-\frac{x}{2 t} \frac{1}{(4 \pi t)^{\frac{n}{2}}} \exp \left(-\frac{|x|^{2}}{4 t}\right) .
$$


Setting $y=\frac{|x|}{\sqrt{|t|}}$, we have

$$
\begin{aligned}
|\nabla G(x, t)|(|x|+\sqrt{|t|})^{n+1} & \leq \frac{|x|}{2(\sqrt{|t|})^{n+2}} \exp \left(-\frac{|x|^{2}}{4 t}\right)(|x|+\sqrt{|t|})^{n+1} \\
& \leq y(1+y)^{n+1} \exp \left(-\frac{|y|^{2}}{4}\right) .
\end{aligned}
$$

Since

$$
\lim _{y \rightarrow \infty} y(1+y)^{n+1} \exp \left(-\frac{|y|^{2}}{4}\right)<+\infty
$$

and

$$
\delta((x, t),(0,0)) \leq|x|+\sqrt{|t|} \leq 2 \delta((x, t),(0,0)),
$$

the conclusion follows.

Proof of Theorem 1.5. Similar to the proof of Theorem 1.4, we divide it into two claims.

Claim 3. $\nabla u \in M^{2,2-2 \alpha}\left(P_{\frac{r_{0}}{2}}\left(x_{0}\right)\right)$ for any $\alpha \in(0,1)$.

To see it, let $P_{r}\left(x_{1}, t_{1}\right) \subset P_{r_{0}}\left(x_{0}, t_{0}\right)$ and $v: P_{r}\left(x_{1}, t_{1}\right) \rightarrow \mathbb{R}^{m}$ solve

$$
v_{t}-\Delta v=0 \text { in } P_{r}\left(x_{1}, t_{1}\right), v=u \text { on } \partial_{p} P_{r}\left(x_{1}, t_{1}\right),
$$

where $\partial P_{r}\left(x_{1}, t_{1}\right)=\left(B_{r}\left(x_{1}\right) \times\left\{t_{1}-r^{2}\right\}\right) \cup\left(\partial B_{r}\left(x_{1}\right) \times\left[t_{1}-r^{2}, t_{1}+r^{2}\right]\right)$ denotes the parabolic boundary of $P_{r}\left(x_{1}, t_{1}\right)$.

Multiplying both (1.7) and (3.6) by $u-v$, integrating over $P_{r}\left(x_{1}, t_{1}\right)$, we get

$$
\int_{P_{r}\left(x_{1}, t_{1}\right)}|\nabla(u-v)|^{2} \leq C \int_{P_{r}\left(x_{1}, t_{1}\right)}|\nabla u|^{2}|u-v| \leq C \epsilon_{0} \int_{P_{r}\left(x_{1}, t_{1}\right)}|\nabla u|^{2},
$$

where the last inequality follows from the maximum principle of $v$ :

$$
\sup _{P_{r}\left(x_{1}, t_{1}\right)}|u-v| \leq \operatorname{osc}_{P_{r}\left(x_{1}, t_{1}\right)} u \leq \epsilon_{0} .
$$

Hence, for any $\theta \in(0,1)$,

$$
\begin{aligned}
(\theta r)^{n} \int_{P_{\theta r}\left(x_{1}, t_{1}\right)}|\nabla u|^{2} & \leq(\theta r)^{-n} \int_{P_{\theta r}\left(x_{1}, t_{1}\right)}|\nabla v|^{2}+\theta^{-n} r^{-n} \int_{P_{r}\left(x_{1}, t_{1}\right)}|\nabla(u-v)|^{2} \\
& \leq C\left(\theta^{2}+\epsilon_{0} \theta^{-n}\right) r^{-n} \int_{P_{r}\left(x_{1}, t_{1}\right)}|\nabla u|^{2},
\end{aligned}
$$

where we have used the following inequality for $v$ :

$$
(\theta r)^{-n} \int_{P_{\theta r}\left(x_{1}, t_{1}\right)}|\nabla v|^{2} \leq C \theta^{2} r^{-n} \int_{P_{\theta r}\left(x_{1}, t_{1}\right)}|\nabla v|^{2} .
$$

For any $\alpha \in(0,1)$, first choose $\theta=\theta_{0}(\alpha) \in(0,1)$ such that $C \theta_{0}^{2} \leq \frac{1}{2} \theta_{0}^{2 \alpha}$ and then choose $\epsilon_{0}$ such that $C \theta_{0}^{-n} \epsilon_{0} \leq \frac{1}{2} \theta_{0}^{2 \alpha}$. We obtain

$$
\left(\theta_{0} r\right)^{-n} \int_{P_{\theta_{0} r}\left(x_{1}, t_{1}\right)}|\nabla u|^{2} \leq \theta_{0}^{2 \alpha} r^{-n} \int_{P_{r}\left(x_{1}, t_{1}\right)}|\nabla u|^{2} .
$$

Iterating this inequality finitely many times yields

$$
r^{-n} \int_{P_{r}\left(x_{1}, t_{1}\right)}|\nabla u|^{2} \leq C r^{2 \alpha} r_{0}^{-n} \int_{P_{r_{0}}\left(x_{0}, t_{0}\right)}|\nabla u|^{2}, \forall P_{r}\left(x_{1}, t_{1}\right) \subset P_{r_{0}}\left(x_{0}, t_{0}\right) .
$$

This implies Claim 3. 
Claim 4. $\nabla u \in L^{q}\left(P_{\frac{r_{0}}{2}}\left(x_{0}, t_{0}\right)\right)$ for any $1<q<+\infty$.

For simplicity, assume $\left(x_{0}, t_{0}\right)=(0,0)$ and $r_{0}=4$. Let $\eta \in C_{0}^{\infty}\left(P_{4}\right)$ be such that $0 \leq \eta(x) \leq 1, \eta(x) \equiv 1$ on $P_{2}$, and $\left|\eta_{t}\right|+|\nabla \eta|+\left|\nabla^{2} \eta\right| \leq 32$. Set $z=(x, t)$ and $v(z)=u(z) \eta(z)$. Then $v$ satisfies in $\mathbb{R}^{n+1}$ :

$$
v_{t}-\triangle v=F, F \equiv \eta Q(u)(\nabla u, \nabla u)-\left(u-u_{4}\right)\left(\eta_{t}-\triangle \eta\right)-2 \nabla u \nabla \eta,
$$

where $u_{4}=\frac{1}{\left|P_{4}\right|} \int_{P_{4}} u$ is the average of $u$ over $P_{4}$. Then we have

$$
v(z)=\iint_{\mathbb{R}^{n+1}} G(z-w) F(w) d w
$$

so that by Lemma 3.2 we have

$$
|\nabla v(z)|=\left|\iint_{\mathbb{R}^{n+1}} \nabla G(z-w) F(w) d w\right| \leq C I_{1}(|F|)(z) .
$$

Since $F \equiv 0$ outside $P_{4}$, we have $F \in M^{1,2-2 \alpha}\left(\mathbb{R}^{n+1}\right)$ and

$$
\begin{aligned}
\|F\|_{M^{1,2-2 \alpha}\left(\mathbb{R}^{n+1}\right)} & \leq C\|F\|_{M^{1,2-2 \alpha}\left(P_{4}\right)} \\
& \leq C \sup _{P_{r}(z) \in P_{4}}\left\{r^{-2 \alpha-n} \int_{P_{r}(z)}|F(w)| d w\right\} \\
& \leq C\left[\operatorname{osc}_{P_{4}} u+\|\nabla u\|_{M^{2,2-2 \alpha}\left(P_{4}\right)}\right] \leq C .
\end{aligned}
$$

Hence Theorem 3.1 implies that $\nabla v \in L^{\frac{2-2 \alpha}{1-2 \alpha}, *}\left(\mathbb{R}^{n+1}\right)$. Since $\lim _{\alpha \uparrow \frac{1}{2}} \frac{2-2 \alpha}{1-2 \alpha}=+\infty$, we conclude that $\nabla u \in L^{q}\left(P_{2}\right)$ for any $q \in(1,+\infty)$. Now, by the $W_{q}^{2,1}$-estimate for the heat equation, we conclude that $u \in W_{q}^{2,1}\left(P_{1}\right)$ for any $q \in(1,+\infty)$. This, combined with the Sobolev embedding theorem, implies that $\nabla u \in C^{\alpha}\left(P_{1}\right)$ for any $\alpha \in(0,1)$. Now Schauder's theory implies that $u \in C^{\infty}\left(P_{1}\right)$. The proof is now complete.

\section{REFERENCES}

[1] D. R. Adams, A note on Riesz potentials, Duke Math. J. 42 (1975), no. 4, 765-778. MR 0458158 (56:16361)

[2] F. Bethuel, On the singular set of stationary harmonic maps. Manuscripta Math. 78 (1993), no. 4, 417-443. MR.1208652 (94a:58047)

[3] Y. Chen, J. Li, F. H. Lin, Partial regularity for weak heat flows into spheres. Comm. Pure Appl. Math. 48 (1995), no. 4, 429-448. MR1324408 (96e:58039)

[4] Lawrence C. Evans, Partial regularity for stationary harmonic maps into spheres. Arch. Rational Mech. Anal. 116 (1991), no. 2, 101-113. MR.1143435 (93m:58026)

[5] J. Eells, J. Sampson, Harmonic mappings of Riemannian manifolds. Amer. J. Math. 86 (1964), 109-160. MR0164306 (29:1603)

[6] M. Feldman, Partial regularity for harmonic maps of evolution into spheres. Comm. Partial Differential Equations 19 (1994), no. 5-6, 761-790. MR.1274539 (95i:58057)

[7] F. Hélein, Régularité des applications faiblement harmoniques entre une surface et une variété riemannienne (French) [Regularity of weakly harmonic maps between a surface and a Riemannian manifold]. C. R. Acad. Sci. Paris Sér. I Math. 312 (1991), no. 8, 591-596. MR.1101039 (92e:58055)

[8] M. Giaquinta, E. Giusti, The singular set of the minima of certain quadratic functionals. Ann. Scuola Norm. Sup. Pisa Cl. Sci. (4) 11 (1984), no. 1, 45-55. MR752579 (86a:49086)

[9] M. Giaquinta, S. Hildebrandt, A priori estimates for harmonic mappings. J. Reine Angew. Math. 336 (1982), 124-164. MR671325 (84b:58035)

[10] S. Hildebrandt, H. Kaul, K. Widman, An existence theorem for harmonic mappings of Riemannian manifolds. Acta Math. 138 (1977), no. 1-2, 1-16. MR0433502 (55:6478) 
[11] J. Jost, Harmonic mappings between Riemannian manifolds. Proceedings of the Centre for Mathematical Analysis, Australian National University, 4, Australian National University, Centre for Mathematical Analysis, Canberra, 1984. MR756629 (86b:58030)

[12] R. Schoen, Analytic aspects of the harmonic map problem. Seminar on nonlinear partial differential equations (Berkeley, Calif., 1983), 321-358, Math. Sci. Res. Inst. Publ., 2, Springer, New York, 1984. MR765241 (86b:58032)

[13] R. Richard, K. Uhlenbeck, A regularity theory for harmonic maps. J. Differential Geom. 17 (1982), no. 2, 307-335. MR664498 (84b:58037a)

[14] E. M. Stein, Singular integrals and differentiability properties of functions, Princeton University Press, Princeton, New Jersey, 1970. MR0290095 (44:7280)

Department of Mathematics, University of Kentucky, Lexington, Kentucky 40506

E-mail address: thuang@ms.uky.edu

Department of Mathematics, University of Kentucky, Lexington, Kentucky 40506

E-mail address: cywang@ms.uky.edu 